\title{
INTEGRAL/IBIS observations of V404 Cygni polarimetric properties during its 2015 giant flares
}

\author{
P. Laurent ${ }^{1}$ \\ CEA/DRF/IRFU/DAp France \\ E-mail: plaurent@cea.fr \\ C. Gouiffès \\ CEA/DRF/IRFU/DAp France \\ E-mail: christian.gouiffes@,cea.fr \\ J. Rodriguez \\ CEA/DRF/IRFU/DAp France \\ E-mail: jerome.rodriguez@,cea.fr \\ V. Chambouleyron \\ CEA/DRF/IRFU/DAp France
}

V404 Cygni is a low mass X-ray binary believed to host a black hole of 12 solar masses. After 25 years of quiescence, V404 Cygni entered into a period of outburst during which it produced flares largely exceeding its Eddington luminosity. The brightness of the most luminous flares detected with the IBIS telescope has hampered the standard data treatment and produced large telemetry gaps. Polarimetric parameters were thus not measurable with the same data analysis package used with success in the studies of the Crab and Cygnus X-1. We were then forced to develop and implement a special INTEGRAL/IBIS/Compton analysis routine. Our findings show that this new process improved significantly our polarimetry sensitivity not only for very bright flaring events as for V404 Cygni but equally on more standard sources as the Crab nebula. In this paper, we compare the results of our new method with the already published Cygnus X-1 polarization. We show that V404 Cygni were highly polarized (95 $\pm 35 \%$ ) during INTEGRAL revolution 1555 (i.e. 2015 , June $20^{\text {th }}-22^{\text {nd }}$ ) with a polarization angle of $160^{\circ}$, consistent with infrared measurement made at the WHT on June $23^{\text {rd }}$. This achieved sensitivity opens a new window for polarimetric studies with INTEGRAL/IBIS at energies above $200 \mathrm{keV}$.

11th INTEGRAL Conference Gamma-Ray Astrophysics in Multi-Wavelength Perspective 10-14 October 2016

Amsterdam, The Netherlands

\footnotetext{
${ }^{1}$ Speaker

(C) Copyright owned by the author(s) under the terms of the Creative Commons

Attribution-NonCommercial-NoDerivatives 4.0 International License (CC BY-NC-ND 4.0).
} 


\section{Polarimetry with the IBIS/Compton mode}

Polarimetric studies in the hard X-rays/gamma-rays bands are recognized as a powerful tool to better characterize the emission process of high energy sources and their environment. Nevertheless such studies have been hampered by the low flux value of the potential sources and the poor efficiency of the instruments to detect in this range polarized radiation. Compton telescopes, identified since the 1970s as promising $\gamma$-ray polarimeters, encountered several problems as for example the difficulty to remove efficiently the background. The situation changed in 2002 with the launch of the European Space Agency INTEGRAL satellite[1], in particular its IBIS telescope [2] that takes advantage simultaneously of the Compton and coded mask imaging principles. This configuration improves the capabilities of the polarimetric mode and is described in this paper together with results on V404 Cygni.

\subsection{Principles}

The European Space Agency satellite INTEGRAL [1] is dedicated to the deep study of hard $\mathrm{X}$-ray and $\gamma$-ray sources between $20 \mathrm{keV}$ and a few MeV. The mission is based upon the use of two complementary coded-mask telescopes, the imager IBIS [2] and the spectrometer SPI [3]. Both telescopes can also operate as Compton polarimeters. With its double layer of finely pixelated detectors, the IBIS telescope on board the INTEGRAL satellite is well suited for polarimetry studies, between $200 \mathrm{keV}$ and $2 \mathrm{MeV}$. An efficient background subtraction is obtained by deconvolving the coded-mask shadowgram on the primary detector. The angular resolution is energy independent and the two detector planes are close enough to detect events scattered at large angles with a rather uniform response in azimuth.

In a Compton telescope such as IBIS, photons that are Compton scattered between two detectors follow an azimuthal distribution around the source direction that allows quantifying the degree and direction of linear polarization. Indeed, a photon is preferentially scattered in a plane at right angle to its incident electric vector. Photons entering IBIS are Compton scattered in the first detector plane, ISGRI [4], at a polar angle $\theta$ from their incident direction and at an azimuth $\psi$ from their incident electric vector. They are then absorbed in the second detector, PiCsIT [5]. The azimuthal profile $\mathrm{N}(\psi)$, in Compton counts recorded per azimuth bin, follows the relation:

$$
\mathrm{N}(\psi)=\mathrm{S}\left[1+\mathrm{a}_{0} \cos \left(2 \psi-2 \psi_{0}\right)\right]
$$

for a source polarized at an angle PA $=\psi_{0}-\pi / 2+n \pi$ and with a polarization fraction $\mathrm{PF}=\mathrm{a}_{0} / \mathrm{a}_{100}$. The $\mathrm{a}_{100}$ amplitude is expected for a $100 \%$ polarized source. Unfortunately, the IBIS polarimetry capacities have not been calibrated on ground, due to the tight planning of the mission. We have then evaluated $\mathrm{a}_{100}$ to be $0.3 \pm 0.02$ for a Crab-like spectrum between 200 and $800 \mathrm{keV}$, using GEANT3 Monte-Carlo simulations of IBIS and its detailed mass model [6].

Events recorded in ISGRI and PiCsIT within the same time window of $3.8 \mu$ s are tagged as "Compton" events, but do not all result from Compton scattering. Chance coincidences can occur between ISGRI and PiCsIT events independently coming from the source, the sky, or instrumental background. These coincidences are generally called "spurious events". Most of the "Compton"tagged events are due to background events that will be removed by the shadowgram deconvolution; $5 \%$ are due to a small fraction of spurious coincidences that must be removed with high accuracy because they induce false source detection in the sky image. Indeed, among the spurious events, there are some for which the ISGRI or PiCsIT events are really coming from the source: for instance, an ISGRI low energy source signal could be associated by chance with a PiCsIT background event. These events coming from the source are then not removed by the deconvolution process. $2 \%$ come from true Compton events from the source. In the standard process, in order to remove their contribution, the spurious counts are estimated first by computing statistically their 
count rate and then randomizing in time all the ISGRI and PiCsIT events recorded during the observation to produce fake spurious events. Then, we produce shadowgram from these events and subtract them from Compton maps. These maps are therefore deconvolved to remove the background and get source counts. The whole process is applied for events in regularly spaced bins in azimuth to derive $\mathrm{N}(\psi)[7]$.

\subsection{The case of bright sources}

In order to compute correctly the spurious counts contribution, we need a very precise determination of the ISGRI and PiCsIT count rates all over the observation. However, for very bright sources, as V404 Cygni, that showed several flares of fluxes higher than 10 Crabs above $20 \mathrm{keV}$ [8], many telemetry gaps affect the observations (Figure 1 shows the example of flare IV [8] that reached about $43 \mathrm{Crab}$ ), and this hampers a precise determination of the ISGRI count rates. Therefore, using the standard Compton process to treat the V404 Cygni in the presence of telemetry gaps, leads to overestimate the Compton count rate in all energy band, leading, for instance, to an artificial hardening of the spectrum. This stimulated us to search for a new method to recover correctly the Compton events in the specific case of V404 Cygni that could possibly be applied to fainter sources.

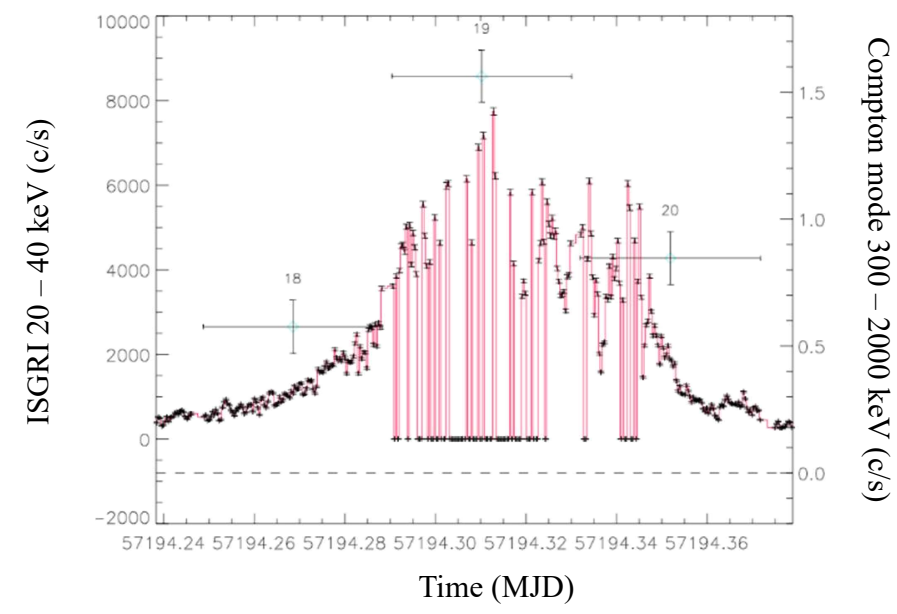

Figure 1: an illustration of ISGRI telemetry losses during the V404 Cygni observation. The right scale gives the ISGRI count rates in bin of 32s; the left scale shows the Compton count rate by science windows. Due to its low count-rate, the Compton mode is not affected by gaps.

(Revolution 1555; science window 18, 19, 20)

\subsection{Toward a new method}

We have consequently searched for a new method able to remove the spurious events contribution without the need of precisely determining the detectors count rates. To do so, we took advantage of the fact that the ISGRI detector records, for each detected event, the pulse height and rise time of the impulsion. The ISGRI rise time is longer for deeper energy deposition inside the $\mathrm{CdTe}$, so low energy - i.e. photoelectric - events have generally a smaller rise time than higher energy, Compton events. This is visible on Figure 2, where we show the rise time distribution of single ISGRI and Compton events, for various science windows over the V404 Cygni observations. As foreseen, it is clearly seen that the ISGRI rise time distribution (in black in Fig. 2) peaks at lower channels than the Compton ones (in green and blue). As mentioned before, during science window 19 (see Figure 1), the ISGRI flux reached a very high level (about $43 \mathrm{Crab}$ at the peak). Thus, it 
caused large telemetry gaps, and induced a large amount of spurious events. This is visible on Figure 2 where the Compton distribution during this observation (red curve) has more low rise time, ISGRI induced, events than a standard Compton distribution (blue and green curve).

We have thus considered using a low threshold in rise time in order to select most of the Compton events while suppressing more than $90 \%$ of the single ISGRI ones. The orange arrow in Figure 2 indicates this threshold. Considering now that no selected events are spurious (which is not formally the case, but we have demonstrated afterwards that the spurious contribution is negligible after this rise time selection), we have no longer to subtract the spurious events in the new analysis.

Of course, this new method has drawbacks as the rise time selection removes also good events and thus decreases the telescope efficiency. However, we do not expect a very strong decrease of the signal to noise ratio, as spurious subtraction in the standard analysis also led to increased uncertainties in the flux determination, an effect that disappears with the new analysis.

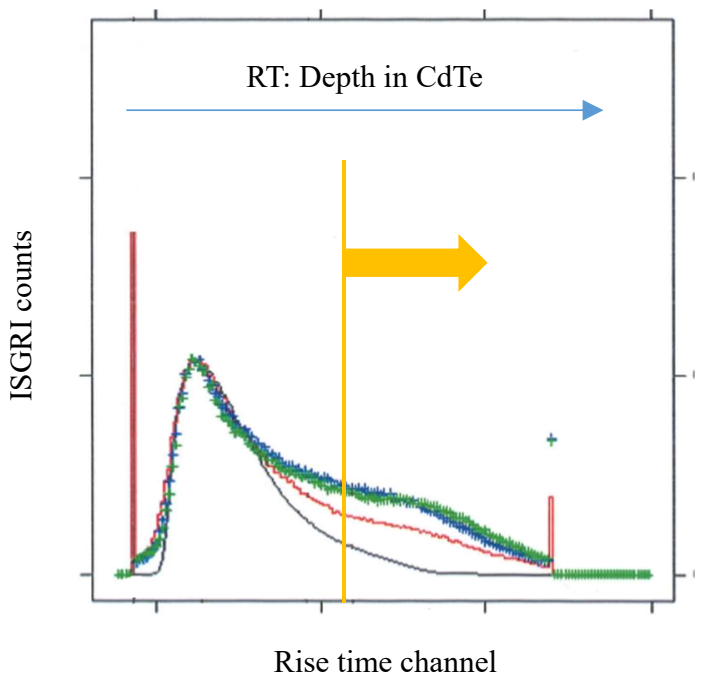

Figure 2: Histogram of the rise time count rate during one science window of rev. 1555 dedicated to V 404 Cygni. Black: ISGRI only events, red: Compton events (scw 19), blue: Compton events (scw 45), green: Compton events ( $\operatorname{scw} 9$ ). The orange arrow indicates the rise time region taken into account in the new data analysis.

\subsection{Verification with Cygnus $\mathrm{X}-1$}

We have first crosschecked that we obtained statistically the same results with this new method as with our previous method, and that removing spurious subtraction does not induce wrong flux determination. We have thus in this context redone the Crab (presented in [9]) and Cygnus X-1 polarization analysis. In the Cygnus X-1 case, we have taken the same data set as in our previous works [10], [11] (Figure 3 - left) and reconstruct with the new method the polarigram (Figure 3 - right).

Both methods give consistent results in terms of polarization angle and fraction (Table 1). We can also notice that, even with a different and stronger selection criteria, this new analysis enables a significant increase of the Compton data signal to noise ratio. This is thus encouraging for the future polarimetric studies with IBIS, even on a short timescale, of sources of bright and moderate intensity. 
Table 1: INTEGRAL/IBIS Cygnus X-1 polarization parameters in the [400-2000] keV band (Errors are at the $90 \%$ confidence limit)

\begin{tabular}{|c|c|c|c|c|}
\hline & Position Angle & $\begin{array}{c}\text { Polarization } \\
\text { fraction }\end{array}$ & S/N & Reference \\
\hline $\begin{array}{c}\text { "standard } \\
\text { analysis" }\end{array}$ & $40 \pm 15^{\circ}$ & $67 \pm 30 \%$ & 16 & {$[10]$} \\
\hline New analysis & $35 \pm 15^{\circ}$ & $45 \pm 15 \%$ & 34 & This work \\
\hline
\end{tabular}
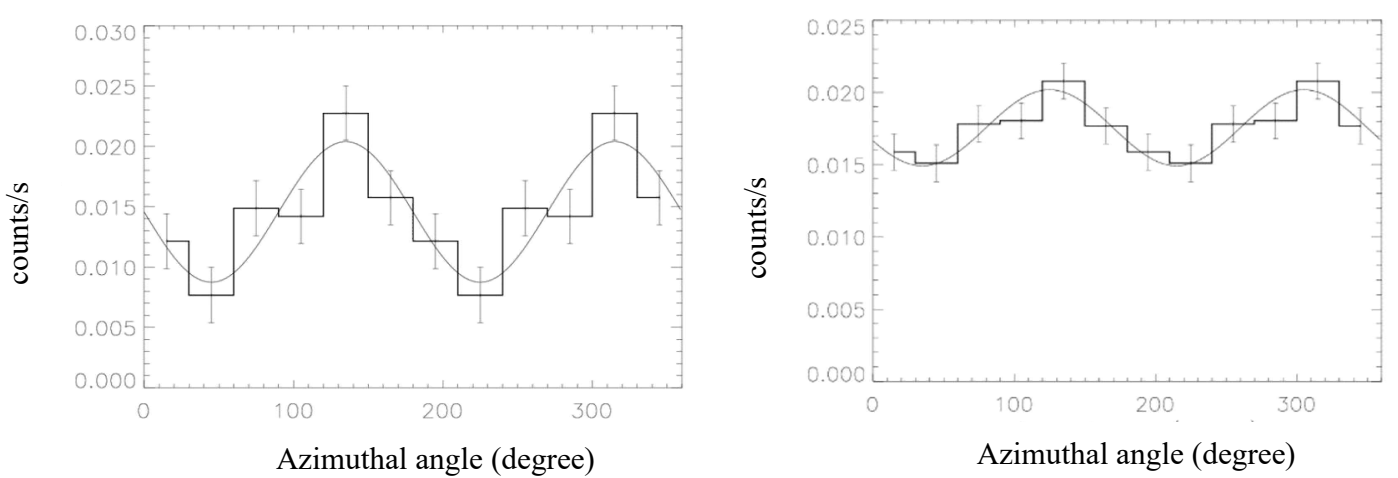

Figure 3: IBIS Polarigrams of Cygnus X-1 obtained in the [400-2000] keV band with the two methods. Left: with the "standard" method [10]. Right: with the new method described in this paper. The two methods give consistent results (see text).

\section{IBIS/Compton spectro-polarimetry of V404 Cygni}

\subsection{V404 Cygni light curve and spectra}

The exceptional 2015 bright flaring activity of V404 Cygni offered a unique opportunity to study into details the polarimetric characteristics of the source. We thus have studied the V404 Cygni spectral and polarimetric properties using the new data analysis software. We have computed the source spectrum taking into account all the Compton events recorded during rev. 1555, with the rise time selection method described above and deconvolved the images in several energy bands to get the source flux.

This new spectrum, between 300 and $2000 \mathrm{keV}$, is given in Figure 4. The preliminary spectral analysis shows that it consistent with a power-law spectrum $\left(d F(E) \propto E^{\wedge}(-\Gamma) d E\right.$ with $\Gamma \sim$ 2.3) with a spectral break around one MeV. However, we cannot determine these parameters precisely, as the new method removes "good" events with low rise time, and our standard Compton response matrices are therefore no longer valid. The computation of these new matrices is underway, and we will be able to characterize quantitatively the spectrum in a near future.

We have also computed the source light curve during rev. 1555, as shown in Figure 5. As the Compton count-rate is much lower that of ISGRI, these data are not affected by telemetry gap problems, and the Compton points are perfectly defined. Figure 5 indicates for most of the time a 
similar evolution of the low energy ISGRI data (20-40 keV) with respect to the higher energy Compton events (300-2000 keV) and that a positive correlation can be established. Nevertheless, for science windows 31 or 38 around MJD 57195 for instance, we can see an anti-correlation between these two energy bands. Again, this comparison is complicated due to the presence of the telemetry gaps. A complete study of this time resolved spectral evolution is beyond the scope of this paper, and reserved for a future dedicated publication.

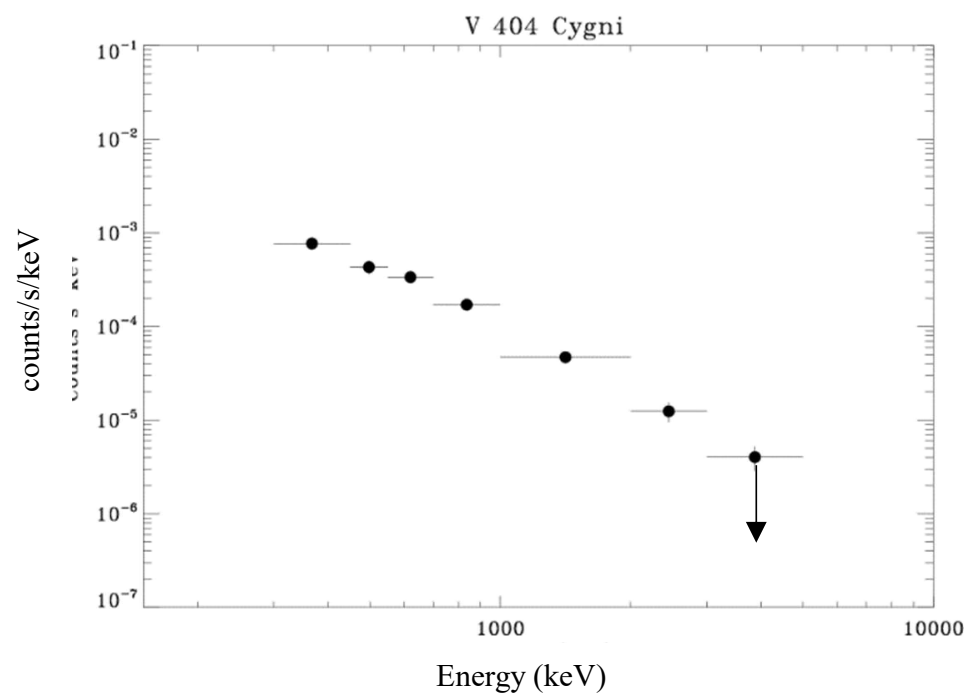

Figure 4: IBIS/Compton count spectrum of V404 Cygni during revolution 1555. The spectrum is consistent with a power law with a break around $1 \mathrm{MeV}$.

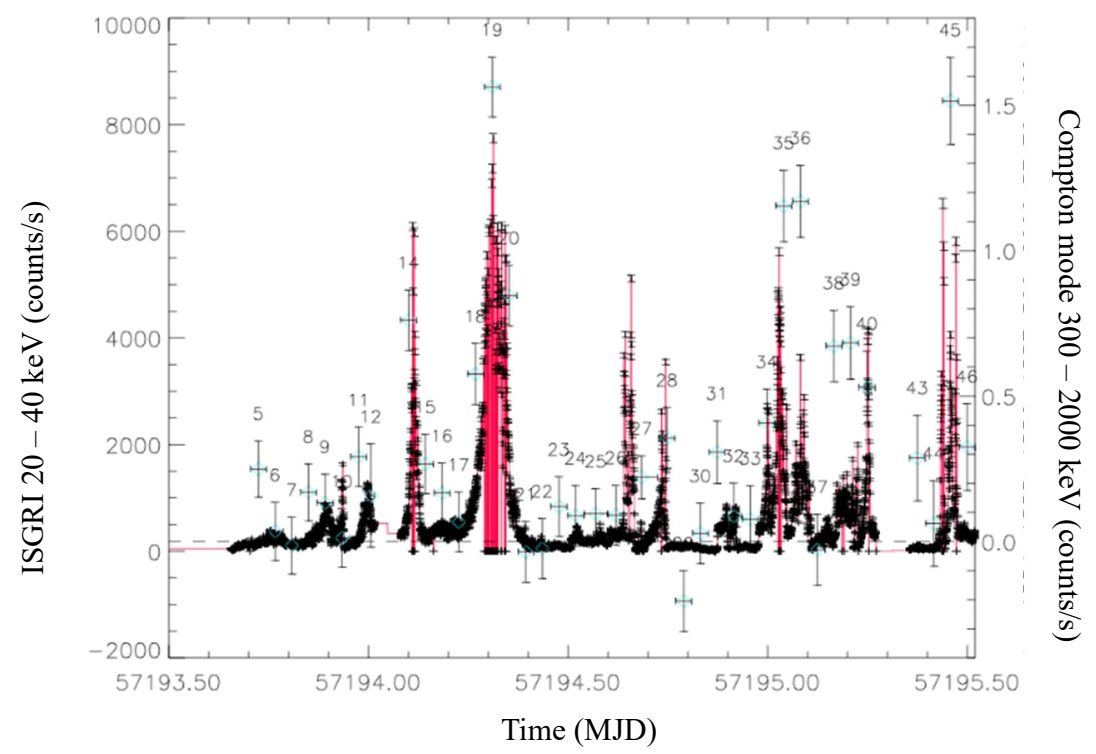

Figure 5: ISGRI and Compton light curves of V404 Cygni during revolution 1555. The ISGRI light curve is made in 32s time bins whereas, due to their lower count rate, Compton events are stacked on science window timescale. Whereas the telemetry loss in ISGRI are clearly seen (see e.g. around MJD 57194.3), the Compton mode is unaffected (See text for details). 


\subsection{Hard X-ray/ $\gamma$-ray Polarization}

Finally, we have computed the V404 Cygni polarigrams, each time taking into account all data recorded during an INTEGRAL revolution. The study of the 10 revolutions dedicated to the source in June 2015 (see Table 2) shows quite surprisingly that polarization is detected only during rev. 1555. Indeed, the source was even brighter in rev. 1557 but we detected no polarization signal at that time $(\mathrm{PF}<17 \%)$. This may imply that the signal is truly non-polarized or that the polarization angle varied during these revolutions. The polarigram obtained during rev. 1555 is shown on Figure 6 (left) and the polarization parameters are given in Table 3.

As it could be seen from Figure 6, our results are consistent with the near infrared (NIR) polarization detection [12]. It is worth noting however that our measurement occurs three days before the NIR measurements, and that we have detected no polarization signal contemporaneously to the NIR data (around INTEGRAL revolution 1556).

Table 2: INTEGRAL/IBIS V 404 Cygni polarization study in the [400-2000 keV] band (June 2015). If we sum all events over a revolution, a clear signal is only visible for rev. 1555. No signal is detected when the source is the brightest (rev. 1557). A more detailed study of the polarization temporal evolution is on-going.

\begin{tabular}{|c|c|c|c|c|c|c|c|c|c|c|}
\hline $\begin{array}{c}\text { Revolution } \\
\text { number }\end{array}$ & 1554 & 1555 & 1556 & 1557 & 1558 & 1559 & 1560 & 1561 & 1562 & 1563 \\
\hline $\begin{array}{c}\text { Exposure time } \\
\text { (ks) }\end{array}$ & 170 & 149 & 84 & 153 & 163 & 171 & 93 & 165 & 174 & 117 \\
\hline Compton S/N & 8.8 & 14 & 4.6 & 28 & -0.4 & -0.2 & -0.05 & 1.26 & 1.75 & 1.09 \\
\hline $\begin{array}{c}\text { Polarization } \\
\text { detected } \\
\text { (yes/no) }\end{array}$ & No & Yes & No & No & No & No & No & No & No & No \\
\hline
\end{tabular}

Table 3: INTEGRAL/IBIS V 404 Cygni polarization parameters in the [450-2000 keV] band measured during revolution 1555 .

(Errors are at the $90 \%$ confidence limit)

\begin{tabular}{|c|c|c|c|c|c|}
\hline Period & $\begin{array}{c}\text { Exposure } \\
\text { time }\end{array}$ & Position Angle & $\begin{array}{c}\text { Polarization } \\
\text { fraction }\end{array}$ & S/N & NIR PA \\
\hline $\begin{array}{c}\text { Rev. 1555 (MJD } \\
57193-57194)\end{array}$ & $149 \mathrm{ks}$ & $160 \pm 15^{\circ}$ & $95 \pm 35 \%$ & 14 & $171^{\circ}[12]$ \\
\hline
\end{tabular}



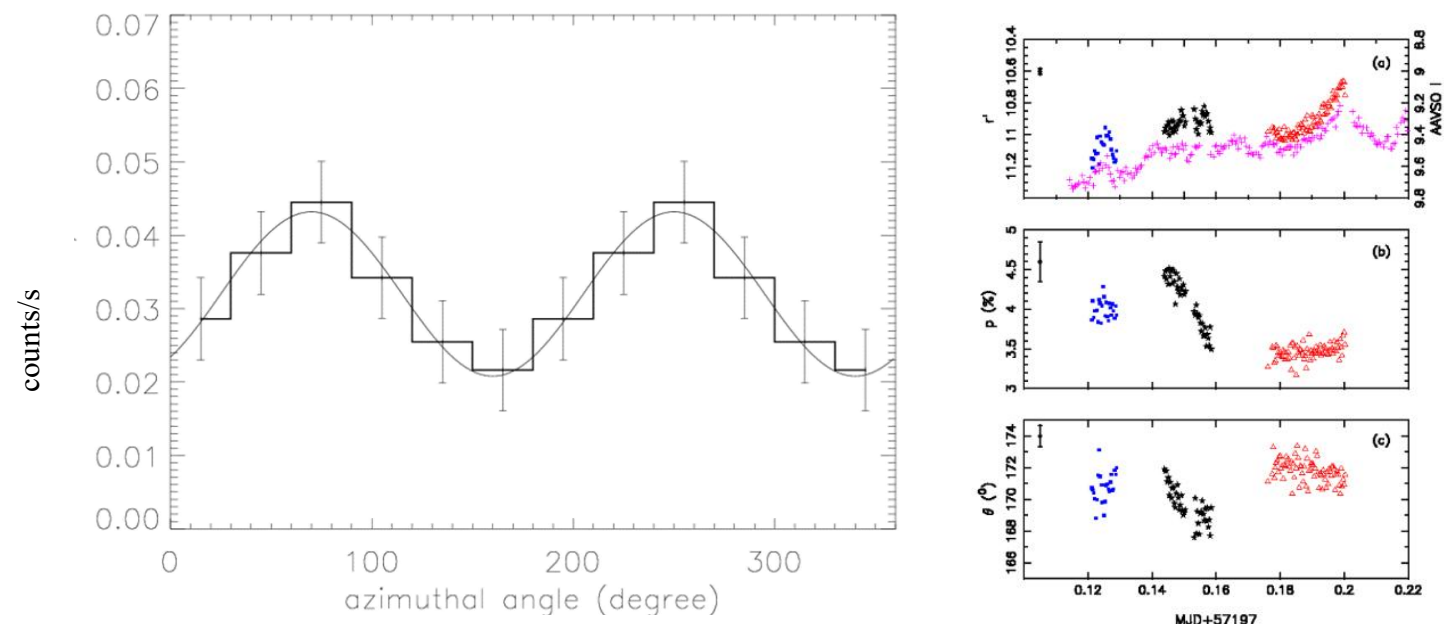

Azimuthal angle (degree)

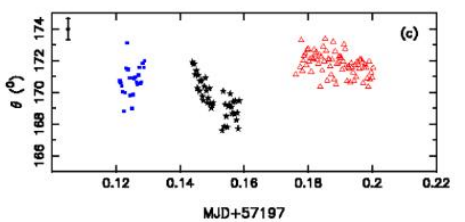

Figure 6: IBIS/Compton polarigram of V404 Cygni computed taking into account all data during rev. 1555 (MJD 57194, left). The polarization angle $\left(\theta=160 \pm 15^{\circ}\right)$ is consistent with the value measured in NIR, three days later $\left(\theta \simeq 171^{\circ}\right.$, right [12]).

\section{Conclusion}

The strong outbursts of V404 Cygni observed in June 2015 have been an excellent and today unique opportunity to study into detail, with the IBIS telescope, the polarimetric properties of a very bright source. Due to the high flux level recorded, a new method based on the rise time profile of the ISGRI signal was implemented. This new analysis allows a better rejection of the spurious events and shows promising results by improving the polarimetric sensitivity of the IBIS telescope. In particular, this method will allow to study the polarimetric behavior of the 2015 outbursts of V404 Cygni on short timescales in correlation with spectral variability/state of the source.

\section{Acknowledgments}

This work is based on observations with INTEGRAL, a European Space Agency (ESA) project with instruments and science data center funded by ESA member states (especially the Principal Investigator countries: Denmark, France, Germany, Italy, Switzerland, and Spain), Czech Republic and Poland, and with the participation of Russia and the United States. The authors acknowledge financial support from the French Space Agency (CNES).

\section{References}

[1] Winkler, C., et al., The INTEGRAL mission, $A \& A, \mathbf{4 1 1}$, L1, 2003

[2] Ubertini, P., et al., IBIS: The Imager on-board INTEGRAL, A\&A, 411, L131, 2003

[3] Vedrenne, G., et al., SPI: the spectrometer aboard INTEGRAL, A\&A, 411, L63, 2003

[4] Lebrun, F., et al., ISGRI: The INTEGRAL Soft Gamma-Ray Imager, A\&A, 411, L141, 2003

[5] Labanti, C., et al., The IBIS-PiCsIT detector onboard INTEGRAL, A\&A, 411, L149, 2003

[6] Laurent, P., et al., Status of the Integral/IBIS telescope modelling and of the response matrices generation, $A \& A, \mathbf{4 1 1}$, L185, 2003

[7] Forot, M., Laurent, P., Lebrun, F. \& Limousin, O., Compton Telescope with a Coded Aperture Mask: Imaging with the INTEGRAL/IBIS Compton Mode, ApJ, 668, 1259, 2007 
[8] Rodriguez J., et al. 2015a, Correlated optical, X-ray, and gamma-ray flaring activity seen with INTEGRAL during the 2015 outburst of V404 Cygni, A\&A, 581, L9

[9] Gouiffès C. et al., New hard X-rays and optical polarimetric observations of the Crab nebula and pulsar, proceeding of the INTEGRAL 2016 conference, PoS(INTEGRAL2016)038

[10] Laurent P., et al., Polarized Gamma-Ray Emission from the Galactic Black Hole Cygnus X-1, Science, 332, 438, 2011

[11] Rodriguez J. et al. 2015b, Spectral State Dependence of the 0.4-2 MeV Polarized Emission in Cygnus X-1 Seen with INTEGRAL/IBIS, and Links with the AMI Radio Data, ApJ, 807, 17

[12] Shahbaz et al., Evidence for magnetic field compression in shocks within the jet of V404 Cyg, MNRAS, 463, 1822, 2016 